Service social

\title{
Les approches intégrées dans l'histoire du service social latino-américain : analyse de trois modèles
}

\section{Ruben Michea}

Volume 36, numéro 2-3, 1987

Approches intégrées

URI : https://id.erudit.org/iderudit/706372ar

DOI : https://doi.org/10.7202/706372ar

Aller au sommaire du numéro

Éditeur(s)

École de service social de l'Université Laval

ISSN

1708-1734 (numérique)

Découvrir la revue

Citer cet article

Michea, R. (1987). Les approches intégrées dans l'histoire du service social latino-américain : analyse de trois modèles. Service social, 36(2-3), 434-453. https://doi.org/10.7202/706372ar
Résumé de l'article

L'auteur montre d'abord comment le contexte des années soixante-dix en Amérique latine, marquée surtout par des conditions de dépendance externe, a provoqué un processus de réflexion, d'analyse et de reconceptualisation du service social latino-américain.

Les milieux de pratique et de formation ont participé à ce mouvement, et Michea fait une présentation descriptive de trois propositions d'approches et de méthodologies de base qui ont marqué l'histoire du service social d'Amérique du Sud. 


\section{Les approches intégrées dans l'histoire du service social latino-américain : analyse de trois modèles}

\section{Ruben Michea}

Les expériences relatées dans cet article se sont déroulées au Brésil et au Chili vers la fin des années soixante et le début des années soixante-dix. Le vent de changement qui parcourait l'Amérique latine dans les années précédentes avait ouvert des perspectives nouvelles à toutes les professions et particulièrement à celles œuvrant dans le domaine des sciences sociales. Le service social ne pouvait rester étranger à ce mouvement. Depuis quelques années, une inquiétude généralisée et une insatisfaction croissante se manifestaient déjà dans la profession : telle que structurée, elle ne répondait plus aux besoins de la population. S'imposait de plus en plus, dans la conscience des travailleurs sociaux, la nécessité d'une reformulation idéologique, théorique, méthodologique et pratique dans l'action sociale professionnelle : il fallait répondre, et rapidement.

C'est ainsi que s'est alors amorcé un processus de réflexion sur la nature, les fondements et les méthodes de la profession. Cette démarche a permis de délimiter la profondeur de la crise et a rendu possible l'apparition de nouvelles orientations et approches méthodologiques.

C'est à propos de celles-ci que nous vous présentons ci-après nos réflexions et commentaires, et nous le faisons à titre d'acteur partie et témoin dans ce processus de changement. Nous nous sommes incorporé au mouvement à l'égal de tous les intervenants de cette époque, inspiré par un désir de renouvellement et d'amélioration de notre pratique professionnelle. Rester neutre équivalait à s'écarter du processus : nous avons accepté le débat et le compromis, inspiré par les valeurs humanistes et chrétiennes. Nous avons pris position sur le plan idéologique, cette position étant en accord avec les énoncés d'Éric Fromm (1962). C'est à 
cette période que sont apparues les premières propositions $d$ 'approches intégrées et de méthodologies de base.

Cet article se veut une présentation descriptive de ces approches et une analyse de leur développement et de leur cheminement ; toutefois, il est évident que nous ne pouvons faire un parcours exhaustif de tout ce qui s'est passé à cette époque dans le service social latino-américain. Par conséquent, nous limiterons notre travail à la présentation de trois propositions ou modèles qui, par leur structure et les éléments qu'ils renferment, sont devenus des points marquants dans l'histoire du service social d'Amérique du Sud.

La première de ces propositions qui s'intitulait "La méthodologie de base" a été formulée, en 1969, par un collectif de professeurs de l'École de service social de l'Université catholique du Chili (Collectif de professeurs, 1969). La seconde, le document de Teresopolis, d'abord publié en 1970 (Gomes, Dantas et al., 1974), a été rédigée par un groupe de travailleurs sociaux brésiliens, à la demande du Centre brésilien de coopération et d'échanges en service social. Enfin, la troisième proposition, "Le projet comme modèle d'action sociale" (Alvarino, Pena et Michea, 1974), a été préparée par un groupe de professeurs de l'École de service social de l'Université nationale du Chili, en 1972.

Notre analyse réfère à l'expérience du service social dans deux pays d'Amérique latine: le Chili et le Brésil. Cela n'exclut pas le fait que partout ailleurs se développaient des mouvements de réflexion et apparaissaient de nouvelles approches sérieuses et valables. Pour des raisons de temps et d'espace, il nous a été impossible de faire le parcours de la totalité de ces approches.

Les buts de notre présentation sont d'abord de démontrer que ces expériences font partie de l'histoire du service social latino-américain, qu'elles ont été une réponse à la crise de la profession dans une période particulière de changement concret et qu'encore aujourd'hui, elles gardent une grande valeur de comparaison et éventuellement, d'orientation pour la recherche de nouvelles approches en service social.

\section{Le contexte}

Une analyse de la situation économique et sociale de l'Amérique latine des années soixante permet de constater que le développement plein et intégral, tel que souhaité par l'ensemble de la population, était limité par les conditions de dépendance externe des pays de la région et par les caractéristiques de cette dépendance dans la structure interne de chaque nation. Expliquer ce phénomène est un sujet qui dépasse les 
limites de notre article. Pour des fins de clarification, nous nous bornerons à en présenter les éléments les plus significatifs (Morales, 1968) :

- un fort investissement étranger dans le domaine d'économie de base ou primaire ;

- une industrialisation précaire et lente à démarrer, mais qui a provoqué une migration du secteur rural vers les villes ;

- la population dans les villes comme facteur d'augmentation de la demande de services publics : habitation, services sociaux et santé ;

- la distribution inégale de la richesse : élite jouissant de revenus comparables à ceux des pays industrialisés et majorité insatisfaite, avec un niveau de vie très bas ;

- une marginalisation économique, sociale et culturelle de la majorité de la population.

- un déséquilibre entre les systèmes de production, dont une croissance disproportionnée du secteur tertiaire ;

- une forte présence de l'État dans l'économie et dans la détermination des politiques sociales.

L'incapacité des structures existantes à répondre aux besoins a créé et alimenté un climat d'effervescence et de révolte dans la population qui, graduellement, a commencé à prendre conscience du besoin de changement.

La révolution cubaine, en 1958, est venue renforcer les croyances de ceux qui voyaient la lutte armée comme seule possibilité de changement. Des mouvements révolutionnaires sont apparus dans tous les coins de l'Amérique latine.

Confronté à ce mouvement général, l' "establishment " a répondu également avec une proposition de changement. De cette époque date l'Alliance pour le progrès, système d'aide internationale proposé par les États-Unis pour encourager les pays de l'Amérique latine à développer leurs économies et à améliorer les conditions de vie des secteurs marginaux.

Les pays ont amorcé, à différents degrés, des changements qui se sont traduits par l'augmentation de la participation populaire, la syndicalisation des travailleurs, la réforme agraire, l'alphabétisation ainsi que le regroupement de quartiers urbains.

Chaque modification au niveau social signifiait de nouvelles demandes pour le service social. Toutes les institutions de la société ont ressenti l'impact de cet esprit de changement. Les regroupements de professionnels et les professions, en général, se sont vus forcés de redéfinir leurs rôles face à une réalité nouvelle. 
L'université a adopté une position d'engagement social d'avantgarde dans la recherche de solutions au problème du sous-développement. Les réformes universitaires, amorcées en 1968, ont ouvert des possibilités de réforme à toutes les professions et particulièrement à celles du domaine des sciences sociales. Le "phénomène social" a secoué les institutions et provoqué un climat de discussion et de critique dont personne n'a pu rester à l'écart.

\section{Le service social}

\section{Évolution historique}

Le développement social a comme conséquence presque automatique d'accroître ou de rendre plus évidents les problèmes sociaux. II est alors essentiel que la société puisse compter sur un groupe de professionnels œuvrant dans le domaine de la pathologie sociale de façon à la fois palliative et curative. Ainsi s'explique l'apparition du service social au cours de la première décennie du vingtième siècle, époque pendant laquelle le libéralisme, doctrine alors dominante, a imprégné les positions philosophiques et idéologiques de la profession.

La situation s'est maintenue sans changement jusqu'au milieu des années soixante. À ce moment, comme réponse à tous les mouvements sociaux, a commencé un processus de réflexion, d'analyse et de reconceptualisation du service social latino-américain.

\section{L’idéologie, les principes et les théories}

La série de changements amorcée a contraint le service social à se redéfinir face à la réalité. Il n'était pas facile de répondre aux questions de base : QUI SERVIR ? POURQUOI SERVIR ? et COMMENT SERVIR?

Cependant, malgré de longues discussions, il a fallu tenir compte de l'idéologie dans l'orientation de la profession et dans la détermination du modèle de société pour laquelle cette profession travaille. L'idéologie est présente dans toute action et plus encore dans l'action sociale. Le service social n'a pas échappé à cette règle qui rejoint l'idée développée par Paulo Freire (1967).

Les énoncés qui suivent (Cisneros, 1975) résument la position idéologique du service social traditionnel: 
- le service social avait une forte orientation vers l'assistance comme telle ;

— son objectif était de faciliter l'adaptation de l'individu à la société et à son milieu ;

- l'ensemble des méthodes et techniques avaient comme objet l'individu: tout était orienté vers la personne qui avait un problème et non vers la société.

Les résultats de la réflexion ont contribué au changement d'orientation et la formulation de nouvelles propositions méthodologiques. Un des premiers éléments à considérer était le déplacement du foyer d'intervention de l'individu à la société. Cela ne signifiait nullement ne plus tenir compte de l'individu mais, plutôt, partir de la totalité pour arriver à la particularité. II fallait d'abord agir sur la structure qui empêchait le développement et la participation active de la population.

Un deuxième élément était de rendre les individus conscients de leur réalité et du potentiel de changement que comportaient la réflexion critique et la mobilisation populaire dans la recherche de nouvelles manières de vivre.

Afin de permettre au service social de mieux fonctionner, un double processus s'est amorcé. D'abord, la recherche dans les sciences sociales a permis d'énoncer de nouveaux éléments explicatifs de la réalité ; aussi, suite à de nombreuses insatisfactions, une recherche propre au service social s'est développée avec une rigueur méthodologique plus grande et elle a apporté des résultats qui ont nourri le processus de reconceptualisation.

Finalement, le service social a pris position pour le changement et, dans cette optique, il y a eu un retour à la réalité latino-américaine. Le processus d'aliénation et de dépendance culturelle et intellectuelle avait réussi à faire oublier les racines et à faire croire que les valeurs traditionnelles et la richesse culturelle étaient des données négligeables.

Ces éléments de réflexion rendaient possible l'apparition de nouvelles approches. Un sentiment d'appartenance, un renforcement de l'identité et une volonté d'orientation donnaient un élan au travail de création en service social.

\section{Les approches intégrées}

\section{Le modèle de "La méthodologie de base "}

La première proposition que nous présentons est celle de l'École de service social de l'Université catholique du Chili, publiée en 1969. (Collectif de professeurs, 1969). 
Dans un document de réflexion sur les fondements et la méthodologie du service social, un groupe de professeurs et d'étudiants analysaient la situation et proposaient "La méthodologie de base " en remplacement des méthodes traditionnelles. Le document établissait l'orientation idéologique de la profession, faisait état de l'évolution du service social dans le pays et présentait un nouveau modèle d'intervention. Selon les auteurs, cette méthodologie de base devait être :

- participative, en incorporant acteurs et intervenants dans le processus ;

- formative : en apprenant aux participants une nouvelle démarche en vue de solutionner les problèmes et faciliter la prise de conscience des sujets dans l'action, ce qui permettait l'expression de nouvelles valeurs de vie.

Le service social était habitué à travailler de façon verticale, en imposant un système de travail à sa clientèle et en établissant des programmes à son bénéfice sans consultation préalable. Dans la méthodologie de base, l'on considérait comme essentielle la présence des populations concernées dans chaque étape du processus. Le travail avec les groupes et les communautés impliquait le dialogue et l'échange de connaissances et d'expériences. Cette interaction favorisait une prise de conscience réciproque. D'un côté, la population incorporait de nouveaux éléments pour comprendre sa réalité et sa situation et, de l'autre côté, les professionnels vérifiaient la validité de leur bagage technique et de leur besoin d'ajustement. Dans cette dynamique, on respectait une des propositions de base de Paulo Freire, à savoir que personne n'éduque personne, tout le monde s'éduque ensemble.

Après analyse de la réalité sociale et des problèmes sur lesquels le service social intervenait, les auteurs en sont arrivés aux constats suivants :

- les situations sociales auxquelles doit faire face le service social sont complexes, uniques et dynamiques ;

- dans ces situations, interagissent personnes, groupes et communautés, tous des cibles possibles d'intervention en service social ;

- les travailleurs sociaux ont acquis des connaissances les rendant capables de travailler dans diverses dimensions de la réalité sociale et à différents niveaux, tout en disposant d'une méthodologie appropriée ;

- finalement, les méthodes en service social, bien qu'indépendantes l'une de l'autre, sont toutes issues d'une recherche d'efficacité dans l'action. Il a été facile de constater que les étapes de travail de chaque méthode étaient similaires, orientées et ordonnées par une logique commune. 
Après avoir défini l'orientation du travail social, déterminé des perspectives plus globales et effectué une réorganisation du bagage technique, les auteurs ont proposé une seule méthodologie de base pour le service social. Celle-ci était composée d'étapes communes aux trois méthodes traditionnelles, mais appliquées à des sujets ou cibles d'intervention différents.

La méthodologie était formée des étapes suivantes : investigation significative, interprétation diagnostique, programmation, exécution et évaluation. La figure I illustre ces étapes. À noter que le concept de programmation est ici utilisé dans le sens de "programme d'action " en vue de l'organisation optimale d'un processus. Quant à la seconde figure, elle fournit la structure complète de cette méthodologie de base.

Nous constatons que cette méthodologie diffère des méthodes traditionnelles bien davantage par la cible d'intervention choisie que par la structure même. Le processus de connaissance et de compréhension de la réalité suit les mêmes étapes, quelle que soit l'approche choisie.

Il fallait donc conclure que le problème ne se situait pas dans la méthodologie mais plutôt dans l'adéquation ou l'adaptation de celle-ci à la réalité historique et à l'orientation qu'on voulait lui donner. La méthodologie devenait, en conséquence, un instrument vide que l'on pouvait utiliser pour adapter les individus à leur milieu ou pour promouvoir une révolution.

\section{FIGURE I}

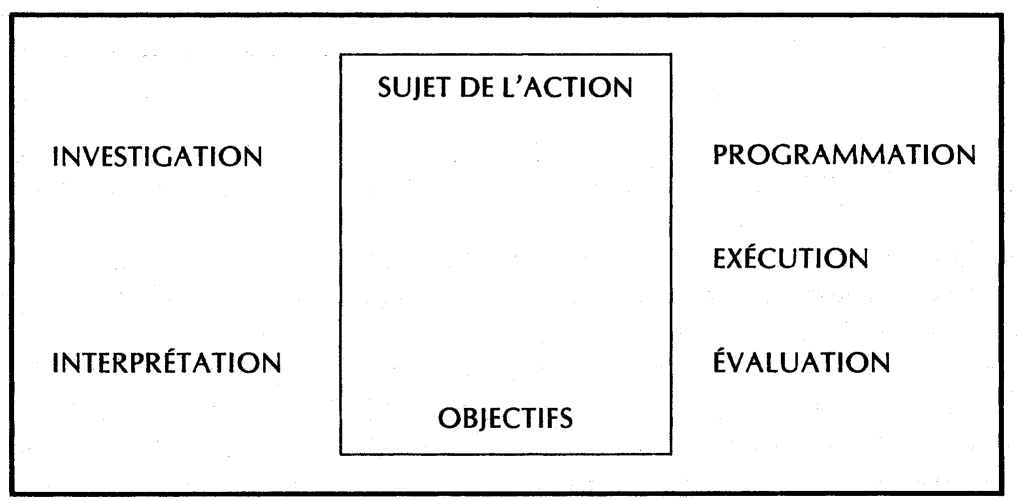




\section{FIGURE II}

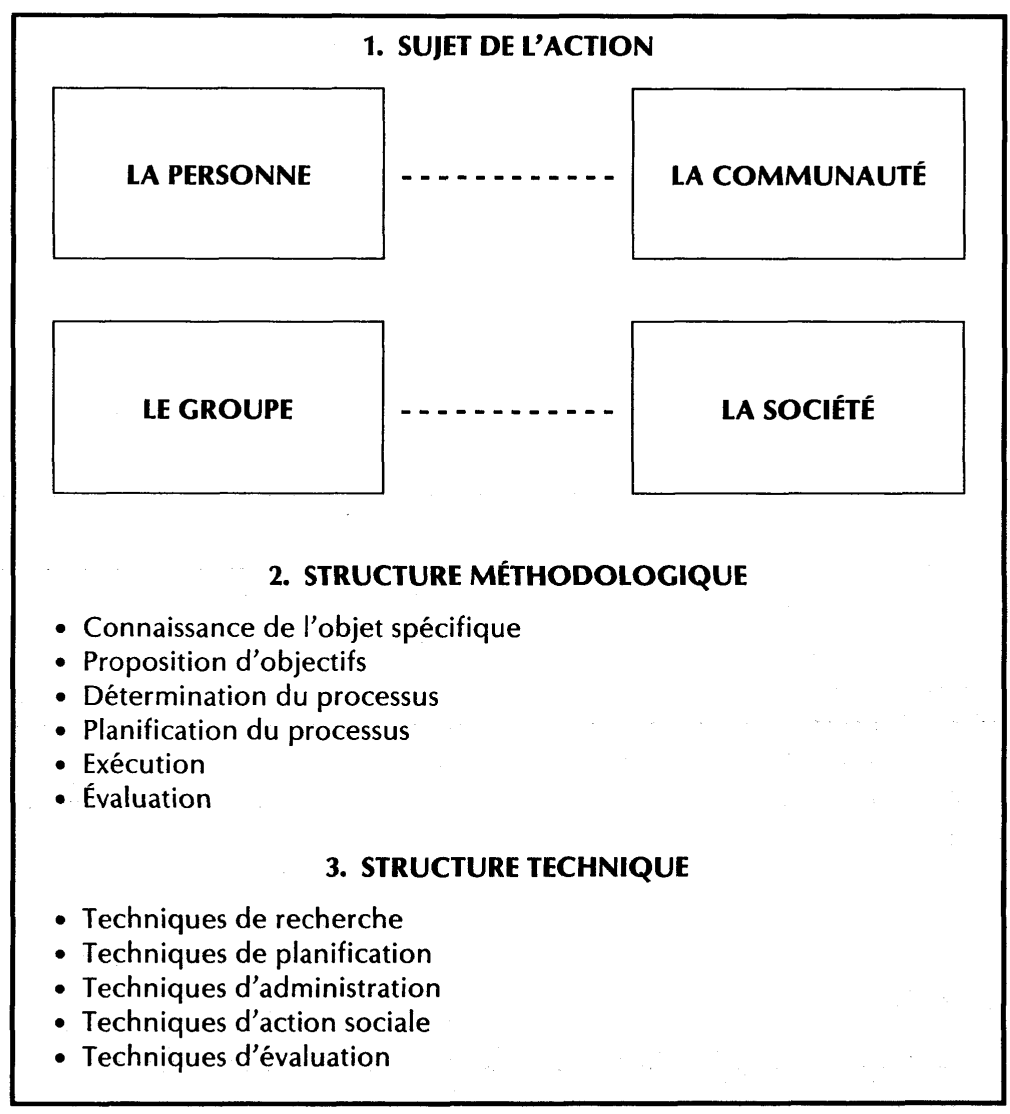

Quelle était, alors, l'originalité de l'approche proposée par l'Université catholique du Chili?

En premier lieu, le changement du foyer d'intervention : un regard sommaire sur la réalité latino-américaine permettait de constater des problèmes structuraux qui affectaient la majorité de la population. L'utilisation de techniques de " casework ", individuelles ou personnalisées, était alors impensable, vu la forte demande. D'ailleurs, les problèmes n'étaient pas des conséquences de l'action individuelle, mais de toute une structure socio-économique qu'il fallait modifier ou changer. 
Deuxièmement, la participation des secteurs concernés, à toutes les étapes du processus : connaissance de leur réalité, prise de conscience du besoin de changement, détermination des priorités et stratégies d'intervention, action ou praxis, recherche de ressources et mobilisation des groupes à cette fin. L'intégration de la population à cette démarche ouvrait de nouveaux horizons. La réflexion de ces divers groupes sur leur réalité allait de la perspective globale à la perspective particulière. Cela permettait de découvrir que le facteur problématique n'était pas néceśsairement le problème mais, plutôt, une manifestation de celui-ci. Cela permettait également de déterminer le degré de difficulté que pouvait présenter la solution d'un problème ainsi que de connaître d'autres difficultés connexes. On pouvait donc établir un plan d'action qui en tienne compte.

Un troisième aspect de cette approche concerne les rôles joués par les travailleurs sociaux dans l'utilisation de cette méthodologie. Aux rôles traditionnels se sont ajoutées de nouvelles fonctions : éducation, animation, planification, promotion et mobilisation sociale, sans oublier cette importante fonction de favoriser la prise de conscience. Ces rôles devaient s'intégrer au processus de changement. L'introduction de nouvelles techniques dans le domaine de l'intervention sociale a favorisé I'incorporation des travailleurs sociaux et des populations au mouvement du changement. Recherche, planification et administration sont des aides précieuses mises à la disposition de la population.

Finalement, avec une perspective plus vaste, les domaines de travail se sont élargis, rejoignant des secteurs jusque-là inconnus pour l'action sociale. Coopératives de paysans, regroupements populaires, syndicats industriels, groupes de femmes, voilà des terrains fertiles pour le travail social. L'élargissement s'est également produit au niveau vertical. Des travailleurs sociaux se sont engagés dans la définition de politiques sociales et dans la formulation des programmes de travail au niveau communautaire. Il faut tout de même préciser que les approches individuelles n'ont jamais été écartées, mais utilisées selon les circonstances et les besoins.

Le travail au niveau de la méthodologie s'est poursuivi et continue encore. Par des approches successives, il y a eu un renforcement conceptuel de la méthodologie. Mais nous ne voulons pas aller ici à la spécificité dans l'application de cette approche, mais seulement en donner un aperçu général. 


\section{Le document de Teresopolis}

Publié pour la première fois en 1970 (Gomes, Dantas et al., 1974), ce document, comme le précédent, était une réponse du service social brésilien à la crise que traversait la profession. Déjà, en 1967, quelques travailleurs sociaux avaient amorcé une réflexion sur le sujet. Les résultats ont été publiés dans le document d'Araxa qui divisait la pratique du service social en deux niveaux : la Macro Action ", comprenant la politique sociale et la planification, et la "Micro Action", incluant l'administration de services et le service direct à la clientèle. Sous le titre "services directs" se situaient les divisions classiques en service social (cas - groupe - communauté). Nous voyons d'emblée que le service social brésilien situait son action à tous les niveaux de la société.

Aucune réflexion n'est possible sans principes de base. Pour l'élaboration de ce modèle, le travail a été guidé par les postulats suivants :

- professionnalisme : le service social possède un domaine de la réalité sur lequel agir, un cadre ou modèle de connaissance pour aborder les phénomènes sociaux cibles de son intervention ;

- relation personne-situation-milieu : de cette interaction constante surgissent des problèmes sociaux, et l'analyse de cette interinfluence permet de choisir la méthodologie adéquate ;

- respect de la personne humaine.

Les travailleurs sociaux brésiliens ont découvert que le cadre théorique et conceptuel du service social contenait des failles et erreurs de définition. Ils en ont situé les causes dans l'absence de recherches, la variété et la complexité des problèmes, la confusion entre objet et client et la catégorisation de la méthodologie.

Le but du service social était de prendre part au processus d'orientation sociale que vit une personne ou un groupe par la recherche de solutions normales aux difficultés ou problèmes sociaux. Ce processus se déclenche à partir du moment où l'homme (personne ou groupe), confronté à une difficulté sociale qui empêche sa réalisation personnelle, et faisant usage de ses propres ressources et de celles offertes par la société, cherche une solution. Les composantes du processus d'orientation sociale sont :

- la connaissance de soi et du monde, la compréhension des causes des difficultés vécues et la conscience des ressources disponibles;

- la réflexion sur les causes des problèmes et sur les possibilités et alternatives de solution;

- l'action ou l'exécution des possibilités choisies. 
Le processus d'orientation sociale est naturel. Quand il se développe correctement, il met en évidence les capacités de l'homme à trouver des solutions aux problèmes. Cependant, ce processus peut être déformé ou bloqué, conduisant l'individu à des solutions insatisfaisantes ou, dans la plupart des cas, à l'absence de solution.

En suivant la démarche initiée par le document d'Araxa, les auteurs nous proposent des méthodologies spécifiques à chaque niveau d'action professionnelle. Pour l'intervention en "Micro Action ", les techniques du cycle méthodologique sont les mêmes que dans l'approche traditionnelle. La spécificité apparaît lorsque l'on adapte telle méthodologie aux objectifs du service social. L'intervention en "Macro Action » implique, pour sa part, la participation à toutes les étapes de la programmation sociale, la formulation d'une méthodologie et d'une stratégie en vue d'élaborer et d'appliquer la politique sociale ainsi que l'établissement de voies de communication avec la population afin de l'intégrer au processus de planification.

Dans le document d'Araxa et le document de Teresopolis, les auteurs reconnaissent vivre encore une période d'imprécision et de mésentente. Le processus se continue. Cependant, l'élément le plus important dans la réflexion des travailleurs sociaux brésiliens a été l'établissement d'un concept d'action pratique qui, partant des niveaux les plus généraux d'intervention, en est arrivé à fournir une description détaillée de toutes les étapes du processus. C'est à partir de la définition des niveaux que la méthodologie est expliquée et développée : planification, administration de services sociaux, et prestation de services directs.

Méthodologie appliquée au niveau de la planification. La planification était définie, dans le document, comme le processus qui oriente la prise de décisions politiques et rationnelles en vue du développement. La planification était conçue comme interdisciplinaire et incluait le service social. Les étapes en étaient la définition de politiques, l'élaboration de programmes, l'implantation des programmes ainsi que le contrôle et l'évaluation de ceux-ci.

Méthodologie appliquée au niveau de l'administration des services sociaux. À ce niveau, les deux éléments de travail étaient le diagnostic (défini comme l'ensemble des opérations de cueillette des données en vue de déterminer les objectifs des programmes et projets) et l'intervention (comprise comme l'ensemble des opérations administratives requises pour l'exécution des programmes et l'évaluation des résultats).

Méthodologie appliquée au niveau de la prestation des services directs. À ce niveau, les opérations sont les suivantes : pour le diagnostic, identification et description, classification, explication et compréhension, 
FIGURE III

\begin{tabular}{|l|l|l|l|}
\hline \multicolumn{1}{|c|}{$\begin{array}{c}\text { Situation telle } \\
\text { qu'observée par } \\
\text { l'intervenant }\end{array}$} & \multicolumn{1}{|c|}{$\begin{array}{c}\text { Variables } \\
\text { significatives pour } \\
\text { le service social }\end{array}$} & $\begin{array}{c}\text { Fonctions } \\
\text { possibles } \\
\text { du service social }\end{array}$ & \multicolumn{1}{|c|}{$\begin{array}{c}\text { Synthèse } \\
\text { des } \\
\text { fonctions }\end{array}$} \\
\hline Analphabétisme & $\begin{array}{l}\text { Difficulté de communication } \\
\text { verbale et écrite, condition- } \\
\text { née par la pensée magique. } \\
\text { Difficulté d'accès à l'informa- } \\
\text { tion. } \\
\text { Mauvaise utilisation des res- } \\
\text { sources. }\end{array}$ & $\begin{array}{l}\text { Développement des pro- } \\
\text { grammes éducatifs de base. } \\
\text { Motivation et incorporation } \\
\text { aux programmes. } \\
\text { Prise de conscience. } \\
\text { Aide et assistance comme élé- } \\
\text { ments de motivation. }\end{array}$ & $\begin{array}{l}\text { Elarification de base. } \\
\text { clance. } \\
\text { Recherche. } \\
\text { Assistance. } \\
\text { Mobilisation. }\end{array}$ \\
\hline
\end{tabular}


prévision des tendances et des projections ; pour l'intervention, préparation de l'action, exécution et évaluation.

Les champs d'intervention du service social ont été également catégorisés. À chaque domaine corresponsaient les variables les plus significatives de chaque problème ainsi que les fonctions ou rôles susceptibles d'être réalisés par l'intervenant social. Les domaines considérés prioritaires étaient les suivants : biologique ( $y$ inclus les équipements sanitaires) ; familial et domestique ; éducationnel ; civique et municipal ; socio-culturel ; logement et sécurité sociale. Un exemple concret tiré du secteur éducationnel peut nous permettre de voir comment était conçue l'intervention.

Il nous apparaît important de faire remarquer que le modèle d'action constituait un premier modèle établissant une typologie tant des diagnostics que des interventions. Le schéma ne peut s'appliquer de façon rigide, mais il faut convenir qu'il devenait une aide précieuse dans la planification des opérations et la distribution des responsabilités. II était beaucoup plus facile de se situer dans un niveau d'action et de déterminer le bagage technique à utiliser. Nous croyons que l'approche des travailleurs sociaux brésiliens était alors l'une des plus modernes, scientifiques et rigoureuses. La recherche réalisée alors par les sciences sociales tenait compte autant des éléments du service social traditionnel que des plus récentes propositions de la science.

Peut-on qualifier l'approche brésilienne d'approche intégrée? Sans hésitation, nous répondons oui. Elle pouvait assumer des caractéristiques différentes pour des réalités différentes. De plus, nous trouvons des éléments d'intégration dans la détermination des problématiques sociales, dans l'utilisation des étapes du cycle méthodologique, dans la vision d'ensemble de toutes les situations problèmes et surtout dans le choix alternatif de techniques et méthodes dépendant du niveau d'intervention et de la problématique considérée.

Il est vrai qu'une telle approche exigeait un renfort dans la formation scientifique du travailleur social, impliquant l'incorporation de nouvelles techniques, mais il est également vrai que la possession d'un bagage plus complet se reflétera toujours par une meilleure pratique. Finalement, il faut indiquer que les méthodes habituelles de service social n'étaient pas écartées ou abolies; elles étaient plutôt incorporées comme des outils ou techniques du processus et susceptibles d'être utilisées lorsque le besoin se faisait sentir. À côté des méthodes courantes, nous trouvons la planification, la recherche pour l'action, l'administration, l'éducation populaire et la mobilisation sociale. 
Ce modèle, encore original aujourd'hui, reflète un dynamisme bien vivant et est orienté vers l'homme et sa condition (groupe et personne, groupe et communauté).

\section{Le projet comme modèle d'action sociale}

En 1972, la recherche se poursuivait ; le mouvement amorcé vers le milieu des années soixante avait laissé des traces importantes dans toutes les actions du service social. C'était une époque d'agitation et de grandes décisions. Le service social latino-américain, qui avait adopté comme orientations le changement des systèmes et la libération de l'homme, se voyait confronté à des propositions de plus en plus radicales qui, toutes, interprétaient l'action sociale comme une action politique partisane. La polarisation était évidente et le ton montait. Chacune des parties essayait de renforcer sa situation en adoptant des positions populistes. Les acteurs de cette confrontation sociale étaient des représentants du peuple et des secteurs marginaux de la population. La lutte des classes était devenue un principe dans l'action de la profession et ceux qui s'y opposaient, des ennemis du peuple. Il était malaisé de maintenir un travail de recherche et de créativité dans les écoles alors que la vie se passait dans les rues! Nonobstant toute cette agitation, des propositions voulant rétablir l'équilibre dans la profession sont apparues. Cette tendance était partagée par des représentants de tous les secteurs qui avaient commencé à prendre conscience que la situation était allée trop loin.

Reportons-nous au contexte d'alors. En 1972, un groupe de professeurs de l'Université nationale du Chili (Alvarino, Pena et Michea, 1974) a commencé un séminaire portant sur le système chilien de sécurité sociale. Ses objectifs étaient d'analyser les politiques de sécurité sociale et de voir comment ces politiques se traduisaient dans l'action concrète. D'ailleurs, dans les institutions de sécurité sociale, un fort groupe de travailleurs sociaux percevaient leurs efforts comme inutiles et inefficaces face à l'ankylose du système.

Ces professeurs ont d'abord défini le service social comme une discipline orientée vers la transformation sociale. La responsabilité de cette fonction de transformation était aussi partagée par d'autres professions. Après avoir fait la critique de l'approche traditionnelle, les auteurs du document présentaient leur proposition comme suit :

Dans son travail, le professionnel fait face à trois différents niveaux de la réalité : les politiques sociales, les institutions ou structures bureaucratiques responsables de traduire la politique en services, et la communauté qui reçoit le produit du fonctionnement des niveaux politique et administratif. 
Partant de ce constat, le professionnel devait orienter ses actions vers les aspects sociaux du développement, mais en incorporant les bénéficiaires réels et potentiels à la prise de décisions. Ce choix implique motivation et formation. Voyons brièvement les trois facettes de cette réalité.

La politique sociale. Dans une perspective large, le premier problème d'une société est le problème politique compris comme la sélection et la hiérarchisation des objectifs de base de la communauté selon l'échelle de valeurs sociales, éthiques et économiques. Le deuxième problème en est un économique, c'est-à-dire l'ajustement des ressources et des moyens alternatifs à des fins multiples.

Le système bureaucratique. Dans le circuit de l'information (définie comme norme ou politique sociale) et à travers la structure institutionnelle, il y a toujours des changements ou des pertes, ce qui modifie I'intention initiale établie par la politique sociale. Les raisons de cette situation varient de l'utilisation de systèmes de travail démodés ou routiniers, aux blocages idéologiques du personnel responsable de traduire la norme en services.

La communauté. Celle-ci est, jusqu'à présent, un acteur passif du processus, c'est-à-dire incapable d'évaluer ou de critiquer la qualité du produit rendu. Le service social se retrouve donc avec une responsabilité nouvelle : motiver et promouvoir l'organisation, former les participants à assumer des rôles plus actifs et critiques dans la planification des politiques et dans la détermination des objectifs.

Dans l'élaboration d'un modèle pour l'action, les auteurs ont introduit la notion de projet conçu comme une séquence d'activités orientées vers la cueillette et l'analyse de l'information nécessaire à la prise de décisions, à l'exécution et à l'évaluation de l'action. Comme on le voit dans la quatrième figure, les étapes du projet sont liées aux principes, à l'action et à l'effet escompté.

La contribution de cette approche se situe surtout dans I'utilisation du concept de modèle de planification et dans la capacité de ce modèle d'incorporer des éléments nouveaux (connaissances et techniques) pour l'adapter à la réalité.

L'approche nous apparaît systémique et, comme les autres approches déjà présentées, part d'une perspective globale et contextuelle pour aboutir à des situations particulières. À leur tour, les éléments particuliers sont analysés et traités à la lumière de cette perspective générale. La structure de la méthodologie est illustrée à la cinquième figure, à partir d'un exemple se référant à la sécurité sociale. 


\section{FIGURE IV}

\begin{tabular}{|c|c|c|}
\hline Principes & Procédures & Conséquences \\
\hline \multicolumn{3}{|l|}{ Étape 1} \\
\hline $\begin{array}{l}\text { Toute action rationnelle doit être } \\
\text { fondée sur la connaissance de la } \\
\text { situation. }\end{array}$ & Investigation & Diagnostic \\
\hline \multicolumn{3}{|l|}{ Étape 2} \\
\hline $\begin{array}{l}\text { La connaissance de la trajectoire d'une } \\
\text { variable et du contexte historique } \\
\text { dans lequel elle se présente permet } \\
\text { de prévoir son comportement futur. }\end{array}$ & Projection & Pronostic \\
\hline \multicolumn{3}{|l|}{ Étape 3} \\
\hline $\begin{array}{l}\text { Connaître la tendance d'une variable } \\
\text { facilite les tentatives de lui donner } \\
\text { une orientation. }\end{array}$ & $\begin{array}{l}\text { Sélection d'un mo- } \\
\text { dèle de référence }\end{array}$ & $\begin{array}{l}\text { Détermination } \\
\text { d'objectifs }\end{array}$ \\
\hline \multicolumn{3}{|l|}{ Étape 4} \\
\hline $\begin{array}{l}\text { La définition claire d'objectifs permet } \\
\text { une prise de décisions rationnelles. }\end{array}$ & Programmation & $\begin{array}{l}\text { Structure de déci- } \\
\text { sions }\end{array}$ \\
\hline \multicolumn{3}{|l|}{ Étape 5} \\
\hline $\begin{array}{l}\text { L'arrangement, la synchronisation et } \\
\text { la chronologie des activités du projet } \\
\text { favorisent sa mise en marche et l'effi- } \\
\text { cacité dans l'action. }\end{array}$ & $\begin{array}{l}\text { Direction et exé- } \\
\text { cution du projet }\end{array}$ & Pratique sociale \\
\hline \multicolumn{3}{|l|}{ Étape 6} \\
\hline $\begin{array}{l}\text { La différence entre la situation initiale } \\
\text { et la situation finale est l'accomplis- } \\
\text { sement du projet. }\end{array}$ & $\begin{array}{l}\text { Recherche éva- } \\
\text { luative }\end{array}$ & $\begin{array}{l}\text { Vérification du } \\
\text { changement }\end{array}$ \\
\hline \multicolumn{3}{|l|}{ Étape 7} \\
\hline $\begin{array}{l}\text { Toute connaissance issue de la pra- } \\
\text { tique sociale doit être utilisée pour } \\
\text { améliorer la situation actuelle et la } \\
\text { théorie disponible. }\end{array}$ & $\begin{array}{l}\text { Systématisation } \\
\text { et orientation de } \\
\text { la nouvelle con- } \\
\text { naissance }\end{array}$ & $\begin{array}{l}\text { Apport de l'ac- } \\
\text { tion à la théorie } \\
\text { et reformulation } \\
\text { de la politique } \\
\text { sociale }\end{array}$ \\
\hline
\end{tabular}




\section{FIGURE V}

\section{Structure de la méthodologie}

\section{Diagnostic global}

\begin{tabular}{|l|l|l|}
\multicolumn{1}{l}{ Schéma des données } & \multicolumn{1}{c}{ Schéma instrumental } & \multicolumn{1}{c|}{ Schéma théorique } \\
\hline $\begin{array}{l}\text { Réalité nationale. Sé- } \\
\text { curité sociale. }\end{array}$ & $\begin{array}{l}\text { Entrevues. Bibliogra- } \\
\text { phie. Analyse de conte- } \\
\text { nu. }\end{array}$ & $\begin{array}{l}\text { Capitalisme. Dépen- } \\
\text { dance. Sous-dévelop- } \\
\text { pement. Doctrine de } \\
\text { la sécurité sociale. }\end{array}$ \\
\hline
\end{tabular}

\section{Diagnostic spécifique}

\begin{tabular}{|l|l|l|}
\multicolumn{1}{|c}{ Schéma des données } & \multicolumn{1}{c}{ Schéma instrumental } & \multicolumn{1}{c|}{ Schéma théorique } \\
\hline $\begin{array}{l}\text { Agence de sécurité so- } \\
\text { ciale. Service social }\end{array}$ & $\begin{array}{l}\text { Entrevues. Observa- } \\
\text { tion. Enquête. }\end{array}$ & $\begin{array}{l}\text { Principes administra- } \\
\text { tifs. Organisation et } \\
\text { méthodes. }\end{array}$ \\
\hline
\end{tabular}

\section{Modèle d'orientation pour le changement}

Schéma normatif Schéma d'hypothèses Schéma instrumental

\begin{tabular}{|l|l|l|}
\hline $\begin{array}{l}\text { Modèle idéal. Système } \\
\text { unifié. }\end{array}$ & $\begin{array}{l}\text { Formation. Motivation } \\
\text { à la participation. }\end{array}$ & $\begin{array}{l}\text { Programmation sur pro- } \\
\text { jets. Service social per- } \\
\text { sonnel ou de groupe. }\end{array}$ \\
\hline
\end{tabular}

Modèle pour la transformation

Unités d'action

Syndicats. Groupes populaires.
Intervenants

Personneldel'agence. Travailleur social. Avocat.
Techniques

Promotion. Motivation. Éducation populaire. Prise de conscience.

Modèle pour l'utilisation de l'information

\begin{tabular}{|l|l|l|}
\multicolumn{1}{c}{ Évaluation } & \multicolumn{1}{c}{ Orientation } & \multicolumn{1}{c|}{ Systématisation } \\
\hline Mesure avant-après. & $\begin{array}{l}\text { Direction. Bureau de } \\
\text { planification. }\end{array}$ & Rapport final. \\
\hline
\end{tabular}




\section{Conclusion}

Les approches intégrées et la reconceptualisation sont apparues en Amérique latine comme une réponse à la crise plus générale de la structure socio-économique des années soixante. Les temps ont changé et le service social est encore sujet à modifications. La démarche n'est pas encore achevée : l'éclatement des gouvernements populistes et l'apparition brutale des dictatures ont amené quelques auteurs à affirmer la nécessité de développer une méthodologie d'intervention professionnelle susceptible de s'implanter à tout moment dans les limites imposées par les conjonctures sociales et politiques.

Nous avons analysé sommairement trois modèles ou propositions de méthodologies; nous en avons établi les caractéristiques et structures. En conclusion, nous aimerions résumer les éléments communs aux approches qui nous apparaissent comme étant susceptibles de s'appliquer (avec les adaptations nécessaires) à d'autres réalités.

Les modèles présentés faisaient partie d'un processus théorique, méthodologique et opérationnel visant à fusionner tâche professionnelle et réclamations de la population latino-américaine. Par conséquent, l'action était soutenue par une définition idéologique claire. Voilà un des premiers éléments : celui de l'orientation de l'action professionnelle et de l'impact de cette action dans la dynamique sociale. C'est à cause de cette position critique vis-à-vis des structures que le service social est devenu un allié naturel des projets destinés à la transformation de la société.

Un autre aspect de la démarche réside dans le fait que le bagage théorique et conceptuel du service social de cette époque n'était pas clairement défini. Afin de pouvoir formuler une nouvelle approche, il fallait d'abord situer précisément le service social dans le domaine des sciences sociales et établir le cadre conceptuel qui le soutenait. Cette démarche a conduit les théoriciens du service social à une recherche de validation de la profession et à la formulation de nouveaux concepts. L'esprit qui animait cette démarche était d'une rigueur scientifique et méthodologique nouvelle dans la profession.

Les nouvelles approches ont été proposées comme une alternative valable à la carence de ressources et au manque d'adéquation entre la méthodologie traditionnelle et les problèmes des secteurs défavorisés. Elles constituaient une réaction face à une situation qui dépassait les réalités individuelles et avait ses origines dans le contexte plus large de la société.

Le changement de perspective - des aspects généraux aux situations particulières - $a$ été adéquat à notre avis, compte tenu des ressources 
limitées. Un tel changement a offert des possibilités intéressantes pour solutionner les problèmes qui affectaient un fort pourcentage de la population.

Les critères thérapeutiques et cliniques qui s'orientaient exclusivement vers le traitement des maux sociaux par le biais des individus pris isolément et sans tenir compte du contexte, ont laissé place aux critères d'action sociale, lesquels intégraient ces individus dans des groupes faisant partie d'une société. Ce changement de perspective a également permis au service social de s'impliquer et d'agir à tous les niveaux de la réalité sociale.

Un autre élément qui nous apparaît pertinent fut l'incorporation active des intéressés (travailleurs sociaux et clientèles) à toutes les étapes du processus de résolution de problème. La participation était devenue un concept et un action-clé du travail professionnel. La participation des secteurs concernés introduisait dans la méthodologie un caractère de validité et y rendait davantage possible l'atteinte des objectifs proposés.

À notre avis, ces approches intégrées ont apporté au service social latino-américain une coordination accrue, une plus grande rigueur scientifique et l'utilisation de techniques telles que la planification et l'administration. La consolidation des connaissances de base et des habiletés professionnelles a permis au service social des pays concernés d'accéder à des niveaux d'action plus généraux, comme par exemple l'administration de projets sociaux et la définition de politiques.

Finalement, nous croyons que ces approches sont un bon agencement des techniques et des méthodes, mais ne constituent pas, à elles seules, la réponse à une crise des services sociaux. Avant de promouvoir l'intégration, il faut s'assurer que les étapes préliminaires aient été accomplies, à savoir la recherche en service social, la détermination d'orientations, la compréhension du rôle critique et surtout l'établissement d'un modèle de société.

\section{Références bibliographiques}

Alvarino, Pilar, Beatriz Pena et Ruben Michea, «El proyecto como modelo de accion social ", Revista de Servicio Social, no 6, avril 1974.

CISNEROS, Elia, "Una filosofia latino americana de servicio social", Debates sociais. $n^{\circ} 20$, mai 1975.

Collectif de professeurs, Algunas reflexiones sobre fundamentos y metholologia de servicio social, Santiago, École de service social, Université catholique du Chili, 1969. 
Freire, Paulo, Sobre la action cultural, Santiago, Chili, ICIRA, 1967.

Fromm, Eric, Marx y su concepto del hombre, Mexico, Fondo de cultural économico, 1962.

Gomes, Suely, Jose Dantas et al., "Documento de Teresopolis ", Debates sociais, no 4, avril 1974.

Morales, Eduardo, Administracion, algunos puntos basicos, Santiago, Chili, CEPAL, 1968. 